\title{
QUOTIENT AND PSEUDO-OPEN IMAGES OF SEPARABLE METRIC SPACES
}

\author{
PAUL L. STRONG
}

\begin{abstract}
Ernest A. Michael has given a characterization of the regular quotient images of separable metric spaces. His result is generalized here to a characterization of the $T_{1}$ quotient images of separable metric spaces (which are the same as the $T_{1}$ quotient images of second countable spaces). This result is then used to characterize the Hausdorff pseudo-open images of separable metric spaces.
\end{abstract}

1. Introduction. A space is a $k$-space if a subset $F$ is closed whenever $F \cap K$ is closed in $K$ for every compact set $K$. A space is sequential if a subset $F$ is closed whenever no sequence in $F$ converges to a point not in $F$. A space is Fréchet if for each subset $A$ and point $x \in \mathrm{cl} A$, there is a sequence in $A$ converging to $x$. (It is easy to see that first countable spaces are Fréchet, Fréchet spaces are sequential, and sequential spaces are $k$-spaces.)

A network in a space [1] is a collection of subsets $\varphi$ such that given any open subset $U$ and $x \in U$, there is a member $P$ of $\varphi$ such that $x \in P \subset U$. A $k$-network (called a pseudobase by Michael [5]) is a collection of subsets $\varphi$ such that given any compact subset $K$ and any open set $U$ containing $K$, there is a $P \in \varphi$ such that $K \subset P \subset U .{ }^{1}$ A cs-network [4] is a collection of subsets $\varphi$ such that given any convergent sequence $x_{n} \rightarrow x$ and any open set $U$ containing $x$, there is a $P \in \varphi$ and a positive integer $m$ such that $\{x\} \cup\left\{x_{n} \mid n \geqq m\right\} \subset P \subset U$. (Note that any $k$-network is a cs-network, which is in turn a network, and any cs-network $\varphi$ which is closed under finite unions satisfies the property that given any convergent sequence $x_{n} \rightarrow x$ and any open $U$ containing $\{x\} \cup\left\{x_{n} \mid n=1,2, \cdots\right\}$, there is a $P \in \varphi$ such that $\{x\} \cup\left\{x_{n}\right\} \subset P \subset U$.)

Received by the editors March 31, 1971.

AMS 1970 subject classifications. Primary 54C10, 54D55; Secondary 54D50, 54E99.

Key words and phrases. Fréchet spaces, sequential spaces, network, $k$-network, cs-network, $\boldsymbol{\aleph}_{0}$-spaces, sequence-covering mappings, quotient mappings, pseudo-open mappings, separable metric spaces, second countable spaces.

1 Paul O'Meara's definition of a $k$-network (Proc. Amer. Math. Soc. 29 (1971), 183-189) requires only that there be a finite union $R$ of members of $\varphi$ such that $K \subset R \subset U$. Clearly, a space with a countable $k$-network in this sense has one in our sense, and conversely.

(c) American Mathematical Society 1972 
THEOREM 1. The following properties of a $T_{1}$-space $X$ are equivalent:

(a) $X$ is a sequential space with a countable $k$-network.

(b) $X$ is a sequential space with a countable cs-network.

(c) $X$ is a quotient space of a separable metric space.

(d) $X$ is a quotient space of a second countable space.

For a Hausdorff space $X$, these properties are equivalent to

(e) $X$ is a k-space with a countable k-network.

Michael used property (e) to characterize the regular quotient images of separable metric spaces [5, Corollary 11.5, p. 999]. ${ }^{2}$ His proof remains valid for Hausdorff spaces.

THEOREM 2. The following properties of a Hausdorff space $X$ are equivalent:

(a) $X$ is a Fréchet space with a countable k-network.

(b) $X$ is a Fréchet space with a countable cs-network.

(c) $X$ is a pseudo-open image of a separable metric space.

(d) $X$ is a pseudo-open image of a second countable space.

All mappings are assumed to be continuous and onto unless otherwise noted. Many of the ideas used in proving Theorem 1 can be found in [5].

The author would like to thank his teachers, Professors M.-E. Hamstrom and Ross L. Finney, for their advice and encouragement. He is also indebted to the referee, whose suggestions were extremely helpful.

2. Proof of Theorem 1. The following result, which is analogous to [5, Proposition 2.1, p. 984], will be needed.

Proposition 3. If $X$ is a $T_{1}$ sequential space with a countable csnetwork, then $X$ has a countable k-network. In fact, any $T_{1}$ quotient space of $X$ is a sequential space with a countable k-network

PROOF. We prove the second statement, from which the first trivially follows.

Let $f: X \rightarrow Y$ be a quotient mapping where $Y$ is $T_{1}$. Since every quotient space of a sequential space is sequential [2, Proposition 1.2, p. 109], we need only show that $Y$ has a countable $k$-network. Let $\gamma$ be a countable $c s$-network for $X$ which is closed under finite unions. We shall show that $\varphi=\{f(B) \mid B \in \gamma\}$ is a $k$-network for $Y$.

Let $K$ be a compact subset of $Y$ contained in an open set $U$. Let $f\left(B_{1}\right)$, $f\left(B_{2}\right), \cdots$ be the members of $\varphi$ which are contained in $U$, and let $A_{n}=$ $f\left(B_{1}\right) \cup \cdots \cup f\left(B_{n}\right)$. Since $\varphi$ clearly forms a network in $Y$, we know that $K \subset \bigcup\left\{A_{n}\right\}$. We need to show that $K \subset A_{n}=f\left(B_{1} \cup \cdots \cup B_{n}\right)$ for some $n$.

2 Michael calls a reguiar space with a countable $k$-network an $\boldsymbol{\aleph}_{0}$-space. 
Assume the contrary and choose $y_{n}$ in $K-A_{n}$ for each $n$. Since $K \subset \bigcup\left\{A_{n}\right\}$ and since the $A_{n}$ 's are increasing, the set $S=\left\{y_{n}\right\}$ is infinite. Since $K$ is compact, this set has a cluster point $y^{\prime}$ in $K$, so that $S-\left\{y^{\prime}\right\}$ is not closed in $K$, and hence not in $U$. Now $f \mid f^{-1}(U)$ is a quotient mapping since $U$ is open, so $f^{-1}\left(S-\left\{y^{\prime}\right\}\right)$ is not closed in $f^{-1}(U)$. Since open subsets of sequential spaces are sequential [2, Proposition 1.9, p. 110], there exists a sequence $\left\{x_{k}\right\}$ in $f^{-1}\left(S-\left\{y^{\prime}\right\}\right)$ converging to a point $x$ in $f^{-1}(U)-$ $f^{-1}\left(S-\left\{y^{\prime}\right\}\right)$. Since $Y$ is $T_{1}$, each $f^{-1}\left(y_{n}\right)$ is closed, and so the sequence $\left\{x_{k}\right\}$ must contain points from infinitely many of the $f^{-1}\left(y_{n}\right)$. Now $\{x\} \cup\left\{x_{k}\right\}$ is a convergent sequence contained in an open set $f^{-1}(U)$, hence we can find a member $B \in \gamma$ such that $\{x\} \cup\left\{x_{k}\right\} \subset B \subset f^{-1}(U)$. Then $f(B) \subset U$, so $B=B_{n}$ for some $n$. But this implies that $A_{n}$ contains infinitely many of the $y_{n}$ 's, contradicting our choice of them above. This contradiction shows that $\varphi$ is a $k$-network in $Y$.

We shall also use the following result, whose proof is due to Michael [5, Proposition 10.2, p. 994].

Proposition 4 (Michael). $\quad A T_{0}$-space has a countable network if and only if it is a continuous image of a separable metric space.

The following analogues of compact-covering maps and $k(X)$ [5, p. 990] will be used. A mapping $f: X \rightarrow Y$ is sequence-covering [6] if, given any convergent sequence $y_{n} \rightarrow y_{0}$ in $Y$, there exists a convergent sequence $x_{n} \rightarrow x_{0}$ in $X$ such that $f\left(x_{n}\right)=y_{n}, n=0,1,2, \cdots$. Given a space $X$, let $\sigma X[3, \mathrm{p} .52]$ be the set $X$ with topology consisting of the sequentially open $^{3}$ sets of $X$.

The following facts are easily checked:

(1) $\sigma X$ is a sequential space.

(2) $X$ is a sequential space iff $X=\sigma X$.

(3) $X$ and $\sigma X$ have the same convergent sequences.

(4) If $f: X \rightarrow Y$ is continuous, then so is the induced function $f_{\sigma}: \sigma X \rightarrow \sigma Y$. (If $U$ is sequentially open in $Y, f^{-1}(U)$ is sequentially open in $X$.)

The following result is analogous to [5, Lemma 11.2, p. 998] and has a straightforward proof due to Siwiec [6, Theorem 4.1].

Proposition 5. Any sequence-covering mapping whose range is sequential is a quotient mapping.

Finally, we have the following analogue to [5, Theorem 11.4, p. 998].

\footnotetext{
${ }^{3}$ A subset $U$ of $X$ is sequentially open if each sequence in $X$ converging to a point in $U$ is eventually in $U$.
} 
THEOREM 6. The following properties of a $T_{1}$-space are equivalent:

(a) $X$ has a countable cs-network.

(b) $X$ is the image, under a sequence-covering mapping, of a separable metric space.

(c) $\sigma X$ is the image, under a sequence-covering quotient mapping, of a separable metric space.

(d) $\sigma X$ is a quotient space of a separable metric space.

(e) $\sigma X$ is a quotient space of a second countable space.

The proof is analogous to Michael's:

(a) $\rightarrow$ (b). Let $X$ be a $T_{0}$-space with a countable $c s$-network $\varphi$. Let $S=\{0\} \cup\{1 / n \mid n=1,2, \cdots\}$ with topology inherited from the real line. Let $X^{S}$ be the set of mappings from $S$ into $X$ (i.e., the set of convergent sequences in $X$ ) with the compact-open topology. Then $X^{S}$ is a $T_{0}$-space with a countable network. (Letting $\gamma$ be a countable basis for $S$ which is closed under finize unions, the set of finite intersections of sets of the form $\left\{u \in X^{S} \mid u(\mathrm{cl} Q) \subset P\right\}$, where $Q \in \gamma$ and $P \in \varphi$, form a network for $X^{S}$.) Hence, by Proposition 4 , there exists a separable metric space $M$ and a mapping $g: M \rightarrow X^{S}$.

Let $\omega: X^{S} \times S \rightarrow X$ be the evaluation mapping $\omega(u, s)=u(s)$. ( $\omega$ is continuous since $S$ is compact. $)$ It is easily checked that $f=\omega_{\circ}\left(g \times 1_{S}\right)$ is a sequence-covering mapping from $M \times S$ to $X$.

(b) $\rightarrow$ (c). This follows easily from the facts about $\sigma X$ quoted above and from Proposition 5.

(c) $\rightarrow$ (d) $\rightarrow$ (e). Obvious.

(e) $\rightarrow$ (a). By Proposition 3, $\sigma X$ has a countable $c s$-network which, by fact (3) above, is also a countable $c s$-network for $X$.

ProOF of THEOREM 1. All implications except $(\mathrm{e}) \rightarrow$ (a) are either obvious or follow trivially from Theorem 6 or Proposition 3.

(e) $\rightarrow($ a). Let $X$ be a Hausdorff $k$-space with a countable $k$-network $\varphi$. We must show that $X$ is sequential. If $F \subset X$ is not closed, then there is a compact subset $K$ of $X$ such that $F \cap K$ is not closed in $K$. Since $\{K \cap P \mid P \in \varphi\}$ is a countable $k$-network for $K, K$ is a metric space [4, Property C, p. 983]. ( $\left\{\operatorname{int}_{K}(K \cap P) \mid P \in \varphi\right\}$ is a countable basis for $K$.) Since $F \cap K$ is not closed in $K$, there exists a sequence in $F \cap K$ converging to a point in $K-(F \cap K)$. Hence we have a sequence in $F$ converging to a point not in $F$.

3. Proof of Theorem 2. We shall use the following proposition which has an easy proof due to S. P. Franklin (see [2, Proposition 2.3, p. 113]).

Proposition 7. (i) If $f: X \rightarrow Y$ is a quotient mapping and $Y$ is Hausdorff and Fréchet, then $f$ is pseudo-open. (ii) If $X$ is Fréchet and $f: X \rightarrow Y$ is pseudo-open, then $Y$ is Fréchet. 
Proof of TheOREM 2. (a) $\rightarrow$ (b). Obvious.

(b) $\rightarrow$ (c). If $X$ is a Fréchet space with a countable $c s$-network, then, by Theorem 1 , there exists a separable metric space $M$ and a quotient mapping $f: M \rightarrow X$. By Proposition 7, $f$ is pseudo-open.

(c) $\rightarrow$ (d). Obvious.

(d) $\rightarrow$ (a). If $X$ is a pseudo-open image of a second countable space, then $X$ is Fréchet by Proposition 7, and $X$ has a countable $k$-network by Theorem 1.

\section{REFERENCES}

1. A. V. Arhangel'skiĭ, Mappings and spaces, Uspehi Mat. Nauk 21 (1966), no. 4 (130), 133-184= Russian Math. Surveys 21 (1966), no. 4, 115-162. MR 37 \#3534.

2. S. P. Franklin, Spaces in which sequences suffice, Fund. Math. 57 (1965), 107-115. MR 31 \#5184.

3. - Spaces in which sequences suffice. II, Fund. Math. 61 (1967), 51-56. MR 36 \#5882.

4. Joe A. Guthrie, A characterization of $\aleph_{0}$-spaces, Gen. Topology Appl. 1 (1971), 105-110.

5. E. A. Michael, $\boldsymbol{X}_{0}$-spaces, J. Math. Mech. 15 (1966), 983-1002. MR 34 \#6723.

6. Frank Siwiec, Sequence-covering and countable bi-quotient mappings, Gen. Topology Appl. 1 (1971), 143-154.

Department of Mathematics, Bucknell University, Lewisburg, Pennsylvania 17837 